\title{
Metastatic Colorectal Cancer: Evolution of the Response Rate and Survival with Therapeutic Progress
}

\author{
Amira Daldoul ${ }^{1}$, Olfa Gharbi1, Laila Ben Fatma', Imene Chabchoub1 ${ }^{1}$, Sonia Zaied ${ }^{2}$, \\ Khaled Ben Ahmed', Faten Ezzairi', Makrem Hochlef', Imtinene Belaid1, \\ Sami Limem², Slim Ben Ahmed1 \\ ${ }^{1}$ Oncological Medical Department of the University Hospital of Sousse, Sousse, Tunisia \\ ${ }^{2}$ Oncological Medical department of the University Hospital of Monastir, Monastir, Tunisia \\ Email: *Amira.krir@outlook.com
}

Received 27 February 2016; accepted 15 May 2016; published 18 May 2016

Copyright (C) 2016 by authors and Scientific Research Publishing Inc.

This work is licensed under the Creative Commons Attribution International License (CC BY). http://creativecommons.org/licenses/by/4.0/

(c) OP Open Access

\begin{abstract}
Introduction: The therapeutic strategy for metastatic colorectal cancer (mCRC) has evolved greatly in the last two decades. This study aimed to compare patients with mCRC treated at the University Hospital of Sousse (Tunisia) through three periods, in terms of treatment strategy; objective response rate (ORR) to chemotherapy; median overall survival (OS) and prognostic factors conditioning OS. Methods: This was a retrospective study covering the period from January 1994 to December 2011. It involved 401 patients with mCRC divided into three groups: group 1 (G1) including patients treated in the period 1994-1998, group 2 (G2) including patients treated in the period 1999-2005 and group 3 (G3) including patients treated in the period 2006-2011. Results: The ORR after 3 cycles of first line chemotherapy was $33 \%, 50 \%$ and $57.8 \%$ for G1, G2 and G3 respectively. The median OS was 13.8 months for G1, 19 months for G2 and 23 months for G3. The prognostic factors associated with a better OS for G3 were absence of initial tumor complication, normal initial level of carcinoembryonic antigen, normal liver function tests, surgery of the primary tumor, surgery of liver metastases and the ORR after three cycles of first line chemotherapy. Conclusion: The development of the therapeutic strategy, whatever of medical or surgical nature, has led to improved response rates and survival of patients treated for mCRC.
\end{abstract}

\section{Keywords}

Colorectal Neoplasms, Prognosis, Chemotherapy, Metastasis

\footnotetext{
${ }^{*}$ Corresponding author.

How to cite this paper: Daldoul, A., Gharbi, O., Ben Fatma, L., Chabchoub, I., Zaied, S., Ben Ahmed, K., Ezzairi, F., Hochlef, M., Belaid, I., Limem, S. and Ben Ahmed, S. (2016) Metastatic Colorectal Cancer: Evolution of the Response Rate and Survival with Therapeutic Progress. Open Journal of Gastroenterology, 6, 127-135.
}

http://dx.doi.org/10.4236/ojgas.2016.65017 


\section{Introduction}

Colorectal cancer (CRC) is the most common digestive cancer in Tunisia and in the world. Given the absence of a national cancer registry in Tunisia, the incidence of CRC is estimated based on three regional registries. According to the registry of the center of Tunisia, the standardized incidence of colon cancer is 6.5 per 100,000 inhabitants per year in men and 6.1 per 100,000 inhabitants per year in women [1].

In the recent years, the therapeutic advances in the treatment of metastatic CRC (mCRC) have changed its natural history. In fact, the median survival of mCRC patients treated with chemotherapy including 5-Fluoro Uracil (5-FU) was between 6 and 12 months [2], while this median survival after introduction of oxaliplatin, irinotecan and targeted therapies was prolonged up to 30 months, or even more with personalized targeted therapies based on the molecular profile of the tumor [3]. To our knowledge, this evolution has not been assessed over a long period in a same center and such evaluation would give a better idea about the progress resulting from therapeutic advances.

In this context, this study aimed to compare patients with mCRC treated at the University Hospital of Sousse (Tunisia) through three periods (1994-1998, 1999-2005 and 2006-2011) in terms of treatment strategy; objective response rate (ORR) to chemotherapy; median overall survival (OS) and prognostic factors conditioning OS.

\section{Methods}

This was a retrospective study covering the period from January 1994 to December 2011. It involved 401 patients treated in the oncological medical department of the University Hospital of Sousse and fulfilling the following inclusion criteria: histological Proof of rectal or colic adenocarcinoma and metachronous or synchronous metastases of CRC. Evaluation was done every three cycles of chemotherapy. Chemotherapy was continued until tumor progression or occurrence of severe toxicity of chemotherapy.

Chemotherapy protocols used were as follow:

-Fufol: 5-fluorouracil (5FU), $425 \mathrm{mg} / \mathrm{m}^{2} /$ day, day1 to day 5, Folinic acid: $20 \mathrm{mg} / \mathrm{m}^{2} /$ day, day 1 to day 5; simplified LV5FU2: folinic acid $400 \mathrm{mg} / \mathrm{m}^{2}$ in 2 hours and 5FU $400 \mathrm{mg} / \mathrm{m}^{2}$ in $10 \mathrm{~min}$ then $5 \mathrm{FU} 2400 \mathrm{mg} / \mathrm{m}^{2}$ as a continuous infusion of 44 hours;

-Folfiri: irinotecan $180 \mathrm{mg} / \mathrm{m}^{2}+$ simplified LV5FU2;

-Folfox4: oxaliplatin $85 \mathrm{mg} / \mathrm{m}^{2}$ in 2 hours + simplified LV5FU2;

-Folfirinox: 5FU, folinic acid, oxaliplatin and irinotecan,

-Capecitabine: $2500 \mathrm{mg} / \mathrm{m}^{2} / \mathrm{j}, 2$ weeks by 3.

-Bevacizumab-Folfiri: Bevacizumab $5 \mathrm{mg} / \mathrm{kg} / 15$ days + Folfiri

The prognostic factors studied were: age, initial stage, general condition according to world health organization (WHO), initial liver function tests, level of the tumor marker carcinoembryonic antigen (CEA), localization of metastases, type of the first line chemotherapy, radiological response to three cycles and six cycles of first, second and third line of chemotherapy and eventual surgery of the primitive cancer and liver metastases. For some results, we divided the 401 patients into three groups: group 1 (G1) including patients treated in the period 1994-1998, group 2 (G2) including patients treated in the period 1999-2005 and group 3 (G3) including patients treated in the period 2006-2011. Periods' choice was guided by availability of treatment which evolved over time depending on the regulations of the Tunisian country. In fact, marketing authorization was given to irinotecan in 1998, to oxaliplatinin 2002 andto bevacizumab in 2010. According to these dates and practice findings, we considered that the G1 patients were treated mainly with 5FU, the G2 patients were treated mainly with irinotecan and G3 patients were treated with oxaliplatine and to lesser extent with bevacizumab.

The date of inclusion considered was that when histological diagnosis was made by biopsy or surgery if metastases were synchronous, and that when at least one metastasis was confirmed if it was metachronous. Median follow-up was 74 months for the groups 1 and 2 together (G1-2) and 63.55 months for G3.

Statistical analyses were performed using SPSS 18.0 software. Dependent variables examined were the response to first line of chemotherapy and OS. The main determinants of ORR were examined using univariate analysis with chi 2 test for comparison of percentages and t Student test or Anova test for equality of means. OS curves were calculated according to the Kaplan-Meier method and between-group comparisons were performed using the log-rank test. In a multivariate analysis, the variables that are statistically associated with a 0.2 threshold were introduced into cox regression model. For all results, a p value less than 0.05 was considered as statistically significant. 


\section{Results}

\subsection{Clinical and Pathological Characteristics of Patients}

The mean age of patients was 53.1 years. Liver metastases were present in $71.6 \%$ of cases. $26 \%$ of patients had more than one metastatic site and $62.6 \%$ of patients had synchronous metastases. A complete surgery of the primary tumor was done for $85 \%$ of patients in G3 and for $68 \%$ of patients in G1-2. The resection of liver metastases was achieved in $16 \%$ of cases for G1-2 versus $19.6 \%$ for G3. Comparison of the three groups revealed a significant increase in the mean age of patients, a significantly higher proportion of synchronous metastases, a significant longer mean time of occurrence of metachronous metastases, a significantly higher proportion of lung metastases and a significant increase in levels of ACE. The comparison of clinical and pathological characteristics of the three groups is summarized in Table 1.

\subsection{Therapeutic Characteristics}

The first line chemotherapy protocols were FUFOL protocol for 76\% of patients in G1 and FOLFIRI protocol for $83 \%$ of patients in G2. For G3, protocols were FOLFIRI protocol in 50.3\% of cases (associated with bevacizumab in $2 \%$ of cases) and FOLFOX protocol in $29.6 \%$ of cases. The difference between groups was statistically significant $(\mathrm{p}<0.001)$. The comparison of therapeutic characteristics of the three groups is summarized in Table 2.

Table 1. Comparison of characteristics of the three groups of patients.

\begin{tabular}{|c|c|c|c|c|}
\hline & $\begin{array}{c}\mathrm{G} 1 \\
\mathrm{~N}=64\end{array}$ & $\begin{array}{c}\mathrm{G} 2 \\
\mathrm{~N}=136\end{array}$ & $\begin{array}{c}\text { G3 } \\
\mathrm{N}=201\end{array}$ & $\mathrm{P}$ value \\
\hline Mean age (years \pm SD) & $46.7 \pm 12.65$ & $51.6 \pm 13.03$ & $56.3 \pm 13.58$ & $<0.001$ \\
\hline \multicolumn{5}{|l|}{ General condition (WHO), n (\%) } \\
\hline 0 & $43(67)$ & $71(52)$ & $58(28.9)$ & \\
\hline 1 & $20(31)$ & $53(39)$ & $107(53.2)$ & $<0.001$ \\
\hline 2 & $1(2)$ & $5(4)$ & $24(11.9)$ & \\
\hline$\geq 3$ & 0 & $7(5)$ & $12(6)$ & \\
\hline \multicolumn{5}{|l|}{ Metastases, n (\%) } \\
\hline Metachronous & $28(44.3)$ & $82(60.3)$ & $62(30.8)$ & $<0.001$ \\
\hline Synchronous & $36(55.7)$ & $54(39.7)$ & $139(69.2)$ & \\
\hline Mean time of occurrence of metastases (months \pm SD) & $14.1 \pm 12.25$ & $12.5 \pm 12.04$ & $18.9 \pm 13$ & $<0.001$ \\
\hline \multicolumn{5}{|l|}{ Localizations of metastases, n (\%) } \\
\hline Liver & $28(44.1)$ & $71(52)$ & $144(71.6)$ & \\
\hline Lung & $2(3.4)$ & $6(4.6)$ & $45(22.4)$ & $<0.001$ \\
\hline Peritoneum & $15(23.7)$ & $21(15.3)$ & $42(20.9)$ & \\
\hline Ovary & $1(1.7)$ & $2(1.5)$ & $25(12.4)$ & \\
\hline Others & $18(27.1)$ & $36(26.6)$ & & \\
\hline \multicolumn{5}{|l|}{ Normal liver function tests, n (\%) } \\
\hline Yes & $45(70.2)$ & $110(81.3)$ & $136(67.6)$ & 0.1 \\
\hline No & $16(25.5)$ & $23(16.4)$ & $22(10.9)$ & \\
\hline Not specified & $3(4.3)$ & $3(2.3)$ & $43(21.5)$ & \\
\hline \multicolumn{5}{|l|}{ Normal initial level of CEA n (\%) } \\
\hline Yes & $38(60)$ & $80(59.1)$ & $64(31.8)$ & 0.01 \\
\hline No & $26(40)$ & $54(39.9)$ & $92(45.8)$ & \\
\hline Not specified & 0 & $2(1)$ & $45(22.4)$ & \\
\hline
\end{tabular}

N: number of patients, G1: group of patients treated between 1994 and 1998, G2: group of patients treated between 1999 and 2005, G3: group of patients treated between 2006 and 2011, WHO: World Health Organization, SD: standard deviation, CEA: Carcinoembryonic antigen. 
Table 2. Comparison of the therapeutic characteristics of the three groups of patients.

\begin{tabular}{|c|c|c|c|c|}
\hline & $\begin{array}{c}\mathrm{G} 1 \\
\mathrm{~N}=64\end{array}$ & $\begin{array}{c}\mathrm{G} 2 \\
\mathrm{~N}=136\end{array}$ & $\begin{array}{c}\text { G3 } \\
N=201\end{array}$ & $P$ value \\
\hline Surgery of the primary tumor, $\mathrm{n}(\%)$ & $42(66)$ & $94(69)$ & $171(85)$ & $<0.001$ \\
\hline Surgery of liver metastases, n (\%) & $9(14)$ & $24(17.5)$ & $40(19.6)$ & 0.3 \\
\hline \multicolumn{5}{|l|}{ First line chemotherapy, n (\%) } \\
\hline Capecitabine & - & - & $26(12.8)$ & $<0.001$ \\
\hline Fufol & $49(76)$ & - & - & \\
\hline LV5FU2 & $8(13)$ & $16(12)$ & $11(5)$ & \\
\hline Folfiri & - & $113(83)$ & $101(50.3)$ & \\
\hline Folfox & - & $7(5)$ & $60(29.6)$ & \\
\hline Folfirinox & - & - & $1(0.6)$ & \\
\hline Bevacizumab-Folfiri & - & - & $2(1.1)$ & \\
\hline \multicolumn{5}{|l|}{ Evaluation after 3 cycles, n (\%) } \\
\hline OR & $21(33)$ & $68(50)$ & $116(57.8)$ & 0.01 \\
\hline CR & $5(8)$ & $16(11.5)$ & $46(23.1)$ & 0.001 \\
\hline \multicolumn{5}{|l|}{ Evaluation after 6 cycles, n (\%) } \\
\hline OR & $22(35)$ & $68(50)$ & $105(52.1)$ & 0.049 \\
\hline CR & $6(9)$ & $38(28)$ & $21(10.1)$ & $<0.001$ \\
\hline Second line chemotherapy, n (\%) & $17(26.6)$ & 39 (28.7) & 79 (39.3) & \\
\hline Capecitabine & - & $5(14)$ & $18(22.8)$ & 0.02 \\
\hline LV5FU2 & $2(12)$ & - & $2(2.5)$ & \\
\hline Folfiri & - & - & $29(36.7)$ & \\
\hline Folfox & - & $18(46)$ & $27(34.2)$ & \\
\hline Bevacizumab-Folfiri & - & - & $2(2.5)$ & \\
\hline $\begin{array}{c}\text { Evaluation after } 6 \text { cycles, n (\%) } \\
\text { OR }\end{array}$ & - & - & $36(45.5)$ & \\
\hline Third line chemotherapy, n (\%) & $3(4.7)$ & $5(3.7)$ & $30(14.9)$ & $<0.001$ \\
\hline Capecitabine & - & - & $18(60)$ & \\
\hline Folfiri & - & - & $5(16.7)$ & \\
\hline Folfox & - & - & $4(13.3)$ & \\
\hline Bevacizumab-Folfiri & - & - & $1(3.3)$ & \\
\hline Others & - & - & $2(0.6)$ & \\
\hline \multicolumn{5}{|l|}{ Evaluation after 6 cycles, n (\%) } \\
\hline OR & - & - & $7(22.2)$ & \\
\hline
\end{tabular}

N: number of patients, G1: group of patients treated between 1994 and 1998, G2: group of patients treated between 1999 and 2005, G3: group of patients treated between 2006 and 2011, OR: objective response. Fufol: 5-fluorouracil (5FU), 425 mg/m²/day, day1 to day 5, Folinic acid: 20 mg/m²/day, day 1 to day 5; simplified LV5FU2: folinic acid $400 \mathrm{mg} / \mathrm{m}^{2}$ in 2 hours and 5FU $400 \mathrm{mg} / \mathrm{m}^{2}$ in $10 \mathrm{~min}$ then $5 \mathrm{FU} 2400 \mathrm{mg} / \mathrm{m}^{2}$ as a continuous infusion of 44 hours; Folfiri: irinotecan $180 \mathrm{mg} / \mathrm{m}^{2}+$ simplified LV5FU2; Folfox4: oxaliplatin 85 mg/m² in 2 hours + simplified LV5FU2; Folfirinox: 5FU, folinic acid, oxaliplatin and irinotecan, Capécitabine: $2500 \mathrm{mg} / \mathrm{m}^{2} /$ day, 2 weeks by 3.

\subsection{Survival and Prognostic Factors}

The ORR after 3 cycles of first line chemotherapy was 33\%, 50\% and 57.8\% for G1, G2 and G3 respectively with a significant difference between the groups $(\mathrm{p}=0.01)$. Twenty-eight percent of patients received secondline chemotherapy in G1-2 versus 39.3\% in G3 ( $\mathrm{p}=0.02$ ) (Table 2). The median OS was 13.8 months for G1, 19 months for G2 and 23 months for G3 (p < 0.001), with a two-year OS of 35\%, 42\% and 47.5\% for G1, G2 and G3 respectively (Figures 1-3).

Prognostic factors associated with a better OS for G1-2 in univariate analysis were: normal liver function tests, 


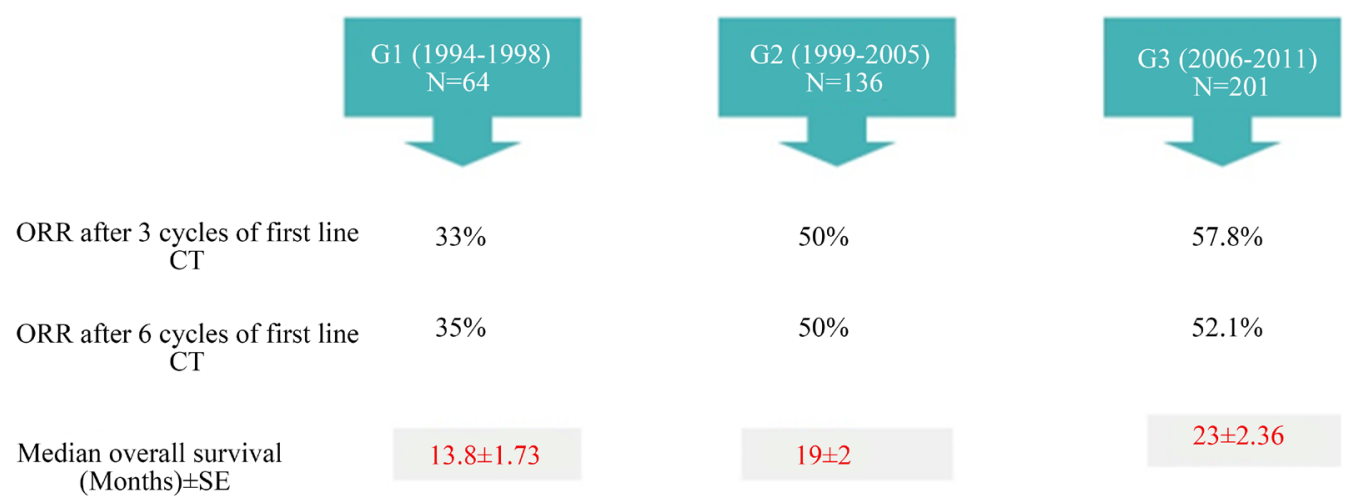

Figure 1. Median overall survival and objective response rate (ORR) to 1st line chemotherapy according to the three groups. N: number of patients, G1: group of patients treated between 1994 and 1998, G2: group of patients treated between 1999 and 2005, G3: group of patients treated between 2006 and 2011, ORR: objective response rate, CT: chemotherapy, SE: standard error of median.

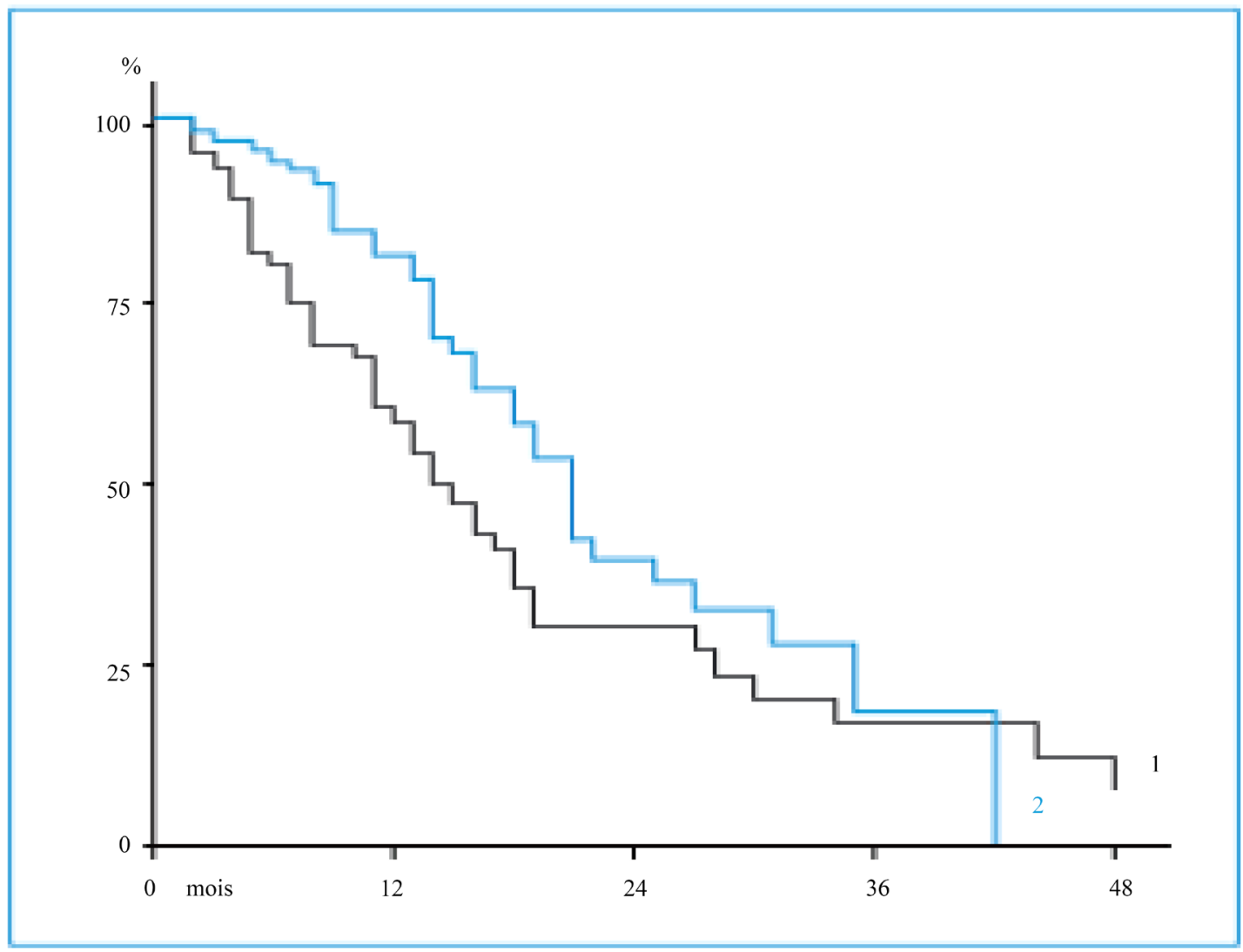

Figure 2. Overall survival curves of groups 1 and 2. 1: patients treated between 1994 and 1998, 2: patients treated between 1999 and 2005, 3: patients treated between 2006 and 2011; horizontal axis represents the number of months.

normal initial level of CEA, surgery of liver metastases and the ORR after three cycles of first line chemotherapy. For G3, prognostic factors of better OS in univariate analysis were: good general condition, absence of initial tumor complication, localization of metastases (no liver and peritoneal localization), normal liver function tests, normal initial level of CEA, surgery of liver metastases, surgery of the primary tumor, the ORR after three cycles of first line chemotherapy and Folfiri \pm bevazicumab as third line chemotherapy (Table 3 ).

In multivariate analyses, prognostic factors for better OS were ORR after 3 courses of first line CT for G1-G2 


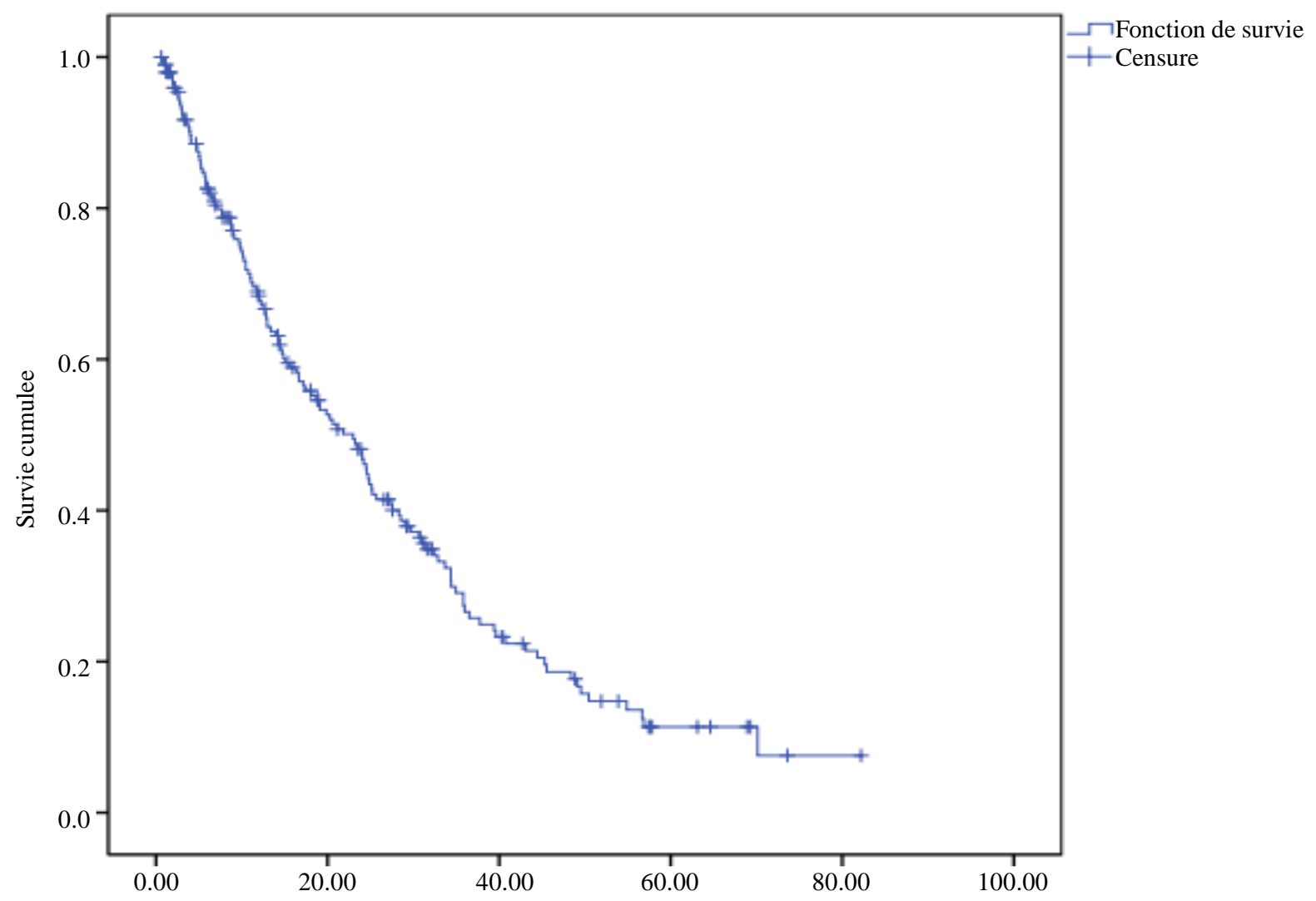

Figure 3. Overall survival curves of group 3: patients treated between 2006 and 2011; horizontal axis represents the number of months.

$(p=0.03)$ and absence of initial tumor complication $(p=0.043)$, surgery of liver metastases $(p=0.002)$ and Folfiri \pm bevazicumab as third line chemotherapy $(p=0.018)$ for $G 3$.

\section{Discussion}

The surgery of the primary tumor is classically indicated for complications of mCRC (tumor bleeding, perforation or obstruction) especially when the complication does not resolve with other therapeutic options (endoscopic stunt, percutaneous embolization, etc.), but its role remains controversial in asymptomatic mCRC [4] [5]. Indeed, there was no significant increase in gastrointestinal complications during chemotherapy for non-operated primary tumors and it has been advanced that if surgery of the primary tumor is avoided, we could spare the patient the morbidity and mortality associated with surgery [6] and delayed start of chemotherapy [7].

In a meta-analysis of 7 uncontrolled studies published in 2010 comparing surgical treatment with chemotherapy for mCRC, the authors concluded that surgery does not reduce the risk of complications from the primary tumor [8]. Despite these data, and although only $5 \%$ to $15 \%$ of patients with mCRC may have a curative surgery for their primary tumor as well as metastasis, up to $75 \%$ of patients undergo surgical resection of their primary tumor [5]. In the present study, surgery of the primary tumor was performed in $68 \%$ of cases for $\mathrm{G} 1-2$ and in $85 \%$ of cases for G3 and it was associated with a significant increase in OS for G3 (p = 0.001). A more recent meta-analysis of 8 retrospective studies published in 2012, resection of the primary tumor was associated with longer survival with a median gain of OS estimated around 6 months [4]. In this study, the gain was estimated at 9 months in G3 (5.88 months versus 15.05 months, $\mathrm{p}=0.001)$. In accordance with these results, in a study by Ferrand et al [5] published in 2013, 72\% of patients were operated with a significant gain in OS (16.3 months versus 19.2 months, $\mathrm{p}<0.0001$ ).

Among patients with liver metastases, only $10 \%$ to $20 \%$ may benefit from surgical resection [9]. In our patients, this rate remains low but tends to increase (16\% for G1-2 versus $19.6 \%$ for G3). This surgery was 
Table 3. Prognostic factors for overall survival in univariate analysis.

\begin{tabular}{|c|c|c|c|c|}
\hline & \multicolumn{2}{|c|}{$\begin{array}{l}\text { G1-G2 (1994-2005) } \\
\mathrm{N}=200 \\
\text { mOS (months) p value }\end{array}$} & \multicolumn{2}{|c|}{$\begin{array}{c}\text { G3 (2006-2011) } \\
\mathrm{N}=201 \\
\text { mOS (months [CI]) p value }\end{array}$} \\
\hline$\geq 2$ & 10.3 & 0.9 & $5.78[1.12-10.44]$ & $<0.0001$ \\
\hline$<2$ & 17.8 & & $24.74[19.87-29.6]$ & \\
\hline \multicolumn{5}{|l|}{ Absence of initial tumor complication } \\
\hline Yes & - & & $28.32[21.83-34.8]$ & 0.038 \\
\hline No & & & $19.84[15.25-24.43]$ & \\
\hline \multicolumn{5}{|l|}{ Metastases } \\
\hline Metachronous & 14.7 & 0.28 & $35.97[29.53-42.42]$ & $<0.0001$ \\
\hline Synchronous & 11.4 & & $13.37[10.73-16]$ & \\
\hline \multicolumn{5}{|l|}{ Localisation of metastases } \\
\hline Liver & 17.6 & 0.3 & 10.65 [12.11 - 21.19] & $<0.0001$ \\
\hline Lung & 18 & & $29.04[5.41-52.67]$ & \\
\hline Peritoneum & 33.2 & & $12.22[9.58-14.86]$ & \\
\hline Ovary & 18.2 & & $15.64[12.55-18.72]$ & \\
\hline \multicolumn{5}{|l|}{ Normal liver function tests (\%) } \\
\hline Yes & 20.5 & $<0.001$ & $24.87[21.12-28.6]$ & 0.007 \\
\hline No & 12.3 & & $10.45[3.6-17.29]$ & \\
\hline \multicolumn{5}{|l|}{ Normal initial level of CEA (\%) } \\
\hline Yes & 24.5 & 0.01 & $33.67[26.6-40.7]$ & $<0.0001$ \\
\hline No & 15.1 & & $18.66[13.36-24]$ & \\
\hline \multicolumn{5}{|l|}{ Surgery of the primary tumor } \\
\hline Yes & - & & 15.05 [11.118.9] & 0.001 \\
\hline No & & & $5.88[4.49-7.27]$ & \\
\hline \multicolumn{5}{|l|}{ Surgery of liver metastases } \\
\hline Yes & 40.7 & 0.05 & $25.2[15.69$ - 34.71] & 0.016 \\
\hline No & 15.1 & & $20.27[13.02-27.52]$ & \\
\hline \multicolumn{5}{|l|}{ ORR after 3 cycles of first line CT } \\
\hline Yes & 15.3 & $<0.0005$ & $29[21.86-36.2]$ & 0.034 \\
\hline No & 13.3 & & $20.2[13.8-26.7]$ & \\
\hline Folfiri \pm bevazicumab as third line CT & - & & $22.75[-]$ & 0.031 \\
\hline
\end{tabular}

N: number of patients, G1-G2: group of patients treated between 1994 and 2005, G3: group of patients treated between 2006 and 2011 , mOS: median overall survival, ORR: objective response rate, CT: chemotherapy.

described as the best treatment allowing a survival rate at 5 years up to $40 \%$ [4]. Similarly in this study, surgery of liver metastases was significantly associated with better OS ( $<0.05$ for G1-2, p = 0.016 for G3).

In the last few years, chemotherapy protocols have multiplied with an increase in terms of efficacy and tolerance. The therapeutic introduction of new cytotoxic agents with effective antitumor activity in mCRC has led to the change of some therapeutic strategies [10]. This study revealed that since 1994, there was a significant improvement in OS with transition of treatment of mCRC from $5 \mathrm{FU}$ alone to its combination with irinotecan oroxaliplatin and with targeted therapies. These survival rates tend to reach those reported in Western studies [11] [12].

Irinotecan and oxaliplatin have completely changed the therapeutic approach of mCRC [10] [13] [14]. The therapeutic strategy is not univocal. Some authors advocate that all patients with mCRC should be given chemotherapy containing oxaliplatin or irinotecan as a first line therapy. Other authors state that if liver metastases 
are never resectable and if the cancer is not very progressive (i.e. not a bulky tumor; minimally symptomatic metastases; conserved general condition with a WHO score of 0 , or if the general condition is impaired, this is due to age or comorbidities), it is possible to initiate treatment with fluoropyrimidine \pm bevacizumab and to establish bitherapy in second-line treatment (escalation therapeutic strategy) [15].

The use of targeted therapies in mCRC is now becoming crucial, especially since improved survival for wild ras tumors up to 33 months [3] has been reported. Two targeted therapies, bevacizumab and cetuximab, had the marketing authorization in Tunisia by 2010 and 2012, respectively. This may explain the low rates of patients having targeted therapy in this study (Folfiri associated with Bevacizumab in $2 \%$ of cases) and incites for the study and analysis of a new period including patients treated with these molecules.

Finally, some limitations need to be noted. In fact, this was a retrospective study, the medical records were incomplete in some cases and the latest news of the patients were not always found. In addition, this study included a period of 12 years, thus creating very heterogeneous groups in terms of treatment options.

\section{Conclusion}

Development of medical and surgical therapeutic strategies resulted in improved survival for patients with mCRC in the center of Tunisia. Within the limits of a retrospective study, our results are comparable to those reported in other studies in terms of therapeutic results and prognostic factors [16]. For the coming years, personalized medicine with treatments targeting the molecular defect and thus allowing avoidance of treatments potentially toxic and ineffective, in addition to progress being made in the fields of genetics, proteomics, medical imaging, and medical therapy, will be challenging but surely beneficial.

\section{Disclosure policy}

The authors declare that there is no conflict of interest regarding the publication of this paper.

\section{References}

[1] Gharbi, O., Chabchoub, I., Limam, S., Hochlef, M., Ben Fatma, L., Landolsi, A., et al. (2010) Prognostic Factors and Survival of Metastatic Colorectal Cancer in the Sousse University Hospital (Tunisia): Comparative Study of Two Treatment Period of 200 Patients. Bulletin du Cancer, 97, 445-451.

[2] Van Cutsem, E., Twelves, C., Cassidy, J., Allman, D., Bajetta, E., Boyer, M., et al. (2001) Oral Capecitabine Compared with Intravenous Fluorouracil plus Leucovorin in Patients with Metastatic Colorectal Cancer: Results of a Large Phase III Study. Journal of Clinical Oncology, 19, 4097-4106.

[3] Heinemann, V., von Weikersthal, L.F., Decker, T., Kiani, A., Vehling-Kaiser, U., Al-Batran, S.-E., et al. (2014) FOLFIRI plus Cetuximab versus FOLFIRI plus Bevacizumab as First-Line Treatment for Patients with Metastatic Colorectal Cancer (FIRE-3): A Randomised, Open-Label, Phase 3 Trial. The Lancet Oncology, 15, 1065-1075. http://dx.doi.org/10.1016/S1470-2045(14)70330-4

[4] Stillwell, A.P., Buettner, P.G. and Ho, Y.H. (2010) Meta-Analysis of Survival of Patients with Stage IV Colorectal Cancer Managed with Surgical Resection versus Chemotherapy Alone. World Journal of Surgery, 34, 797-807. http://dx.doi.org/10.1007/s00268-009-0366-y

[5] Ferrand, F., Malka, D., Bourredjem, A., Allonier, C., Bouché, O., Louafi, S., et al. (2013) Impact of Primary Tumour Resection on Survival of Patients with Colorectal Cancer and Synchronous Metastases Treated by Chemotherapy: Results from the Multicenter, Randomised Trial Federation Francophone de Cancerologie Digestive 9601. European Journal of Cancer, 49, 90-97. http://dx.doi.org/10.1016/j.ejca.2012.07.006

[6] Tebbutt, N.C., Norman, A.R., Cunningham, D., Hill, M.E., Tait, D., Oates, J., et al. (2003) Intestinal Complications after Chemotherapy for Patients with Unresected Primary Colorectal Cancer and Synchronous Metastases. Gut, 52, 568-573. http://dx.doi.org/10.1136/gut.52.4.568

[7] Petrelli, N.J. (2006) Systemic Chemotherapy Should Be the Primary Treatment of Synchronous Colorectal Metastases in the Asymptomatic Patient [Corrected]. Annals of Surgical Oncology, 13, 137-139. http://dx.doi.org/10.1245/ASO.2006.05.059

[8] Cirocchi, R., Trastulli, S., Abraha, I., Vettoretto, N., Boselli, C., Montedori, A., et al. (2012) Non-Resection versus Resection for an Asymptomatic Primary Tumour in Patients with Unresectable Stage IV Colorectal Cancer. Cochrane Database of Systematic Reviews, 8, Cd008997. http://dx.doi.org/10.1002/14651858.cd008997.pub2

[9] Van Cutsem, E., et al. (2014) Metastatic Colorectal Cancer: ESMO Clinical Practice Guidelines for Diagnosis, Treatment and Follow-Up. Annals of Oncology, 25, iii1-iii9. http://dx.doi.org/10.1093/annonc/mdu260 
[10] de Gramont, A., Figer, A., Seymour, M., Homerin, M., Hmissi, A., Cassidy, J., et al. (2000) Leucovorin and Fluorouracil with or without Oxaliplatin as First-Line Treatment in Advanced Colorectal Cancer. Journal of Clinical Oncology, 18, 2938-2947.

[11] Rougier, P., Van Cutsem, E., Bajetta, E., Niederle, N., Possinger, K., Labianca, R., et al. (1998) Randomised Trial of Irinotecan versus Fluorouracil by Continuous Infusion after Fluorouracil Failure in Patients with Metastatic Colorectal Cancer. Lancet, 352, 1407-1412. http://dx.doi.org/10.1016/S0140-6736(98)03085-2

[12] Bouche, O., Scaglia, E. and Lagarde, S. (2010) Prerequisites to the Administration and Prevention of Adverse Effects of Chemotherapy in Colorectal Cancer. Bulletin du Cancer, 97, 265-280.

[13] Douillard, J.Y., Cunningham, D., Roth, A.D., Navarro, M., James, R.D., Karasek, P., et al. (2000) Irinotecan Combined with Fluorouracil Compared with Fluorouracil Alone as First-Line Treatment for Metastatic Colorectal Cancer: A Multicentre Randomised Trial. Lancet, 355, 1041-1047. http://dx.doi.org/10.1016/S0140-6736(00)02034-1

[14] Giacchetti, S., Perpoint, B., Zidani, R., Le Bail, N., Faggiuolo, R., Focan, C., et al. (2000) Phase III Multicenter Randomized Trial of Oxaliplatin Added to Chronomodulated Fluorouracil-Leucovorin as First-Line Treatment of Metastatic Colorectal Cancer. Journal of Clinical Oncology, 18, 136-147.

[15] Koopman, M., Antonini, N.F., Douma, J., Wals, J., Honkoop, A.H., Erdkamp, F.L.G., et al. (2007) Sequential versus Combination Chemotherapy with Capecitabine, Irinotecan, and Oxaliplatin in advanced Colorectal Cancer (CAIRO): A Phase III Randomised Controlled Trial. Lancet, 370, 135-142. http://dx.doi.org/10.1016/S0140-6736(07)61086-1

[16] Wang, H.S., Lin, J.-K., Mou, C.-Y., Lin, T.-C., Chen, W.-S., Jiang, J.-K., et al. (2004) Long-Term Prognosis of Patients with Obstructing Carcinoma of the Right Colon. The American Journal of Surgery, 187, 497-500. http://dx.doi.org/10.1016/j.amjsurg.2003.12.028 